\title{
Plasma cholecystokinin concentrations after breast feeding in healthy 4 day old infants
}

\author{
K Uvnäs-Moberg, G Marchini, J Winberg
}

\begin{abstract}
The aim of the present study was to characterise plasma concentrations of cholecystokinin (CCK) after breast feeding in newborn infants. Fifty eight healthy full term exclusively breast fed infants were investigated at 4 (1) (2-6) days of age. Each infant contributed one blood sample collected just before, immediately after, or 10, 30, and 60 minutes after breast feeding. Plasma concentrations of CCK were measured with a technique consisting of high pressure liquid chromatography separation of gastrins and CCKs and consequent analysis with radioimmunoassay. Mean (SD) preprandial plasma concentrations of CCK (CCK8+CCK-33,39) were 68 (17) $\mathrm{pmol} / 1$. A significant increase was seen immediately after breast feeding, which was followed by a decline at 10 minutes and a secondary rise was seen at 30 and 60 minutes. The first peak is likely to be due to a suckling related activation of the vagal nerve and the second to a stimulatory effect of food on CCK-producing cells. An inverse relationship between basal concentrations of $C C K$ and age of the infant was found. In rats peripheral injections of CCK reduce food intake and cause postprandial sedation and sleepiness via activation of an afferent vagal mechanism. CCK release in response to breast feeding may therefore in addition to exerting stimulatory effects on digestion and metabolism contribute to relaxation and sleepiness seen after breast feeding. The high CCK concentrations seen in younger infants may help the infant to remain satiated and calm despite receiving very little food during the first days of life.
\end{abstract}

(Arch Dis Child 1993;68:46-8)

artment of Pharmacology, Karolinska Institute, Stockholm, Sweden K Uvnäs-Moberg

Department of Paediatrics, Karolinska Hospital, Stockholm, Sweden G Marchini

J Winberg

Correspondence to: Dr Kerstin Uvnäs-Moberg Dr Kerstin UvnasDepartment of Pharmacology, Karolinska Institute, Box 60400, S-104 Stockholm, Sweden.

Accepted 25 June 1992 Cholecystokinin (CCK) is produced in endocrine cells of the proximal intestine. Plasma concentrations have been shown to rise in response to feeding in several species including rats, dogs, and man. ${ }^{1-3}$ The feeding related response is in part caused by a direct effect of nutrients on the endocrine cells. In man, fat seems to be the most important releaser of CCK. ${ }^{4}$ However, vagal nerve activity may contribute to the feeding related response, as in dogs sham feeding stimulates the release of $\mathrm{CCK},{ }^{5}$ and in rats electrical vagal stimulation causes a release of $\mathrm{CCK}{ }^{6}$
We have recently shown that sucking a pacifier in infants causes an increase of plasma concentrations of insulin ${ }^{7}$ and a decrease of the intraluminally secreted somatostatin. ${ }^{8}$ These results indicate that sucking triggers an activation of the vagal nerves which in turn influence the secretion rate of vagally controlled hormones. ${ }^{9}$

CCK is known to stimulate gall bladder contraction and also pancreatic growth and enzyme secretion. ${ }^{10}$ In addition, it has been shown that CCK released from the intestine, via an afferent vagal mechanism, induces satiety and feeding associated sedation and sleepiness. ${ }^{11} 12$

The aim of the present study was to measure plasma concentrations of CCK during the first days of life and to characterise the response during breast feeding. Samples were collected at different time points during and after a breast meal in order to obtain evidence of separate vagal and food related regulatory effects on CCK release.

\section{Subjects and methods}

Fifty eight healthy infants, 30 boys and 28 girls, were studied with the approval of the local medical ethics committee and with consent from the parents. They were all born after uncomplicated pregnancies and at term. Their mean (SD) gestational age and birth weight were 40 (1) weeks and 3490 (405) g, respectively. All infants were exclusively breast fed. Blood samples were collected at 4 (1) days of age (range 2-7 days) in connection with breast feeding. The feed took place 3 (1) hours after the preceding feed and was demanded by the infant according to the mother's evaluation. The infants were allowed to suck to satiety and the feed duration was 27 (10) minutes. The amount of milk ingested, determined by weighing the infants $(n=50)$ before and after a meal, was $50(20) \mathrm{ml}$.

Each infant contributed one blood sample, which was taken just before breast feeding $(\mathrm{n}=12)$, immediately after breast feeding was ended $(n=12)$ or $10,(n=13), 30(n=9), 60$ $(n=12)$ minutes later. Samples of approximately $2 \mathrm{ml}$ venous blood were taken from the back of the hand with an open needle technique in connection with routine metabolic screening. Blood samples were collected into ice chilled plastic tubes containing $10 \mathrm{IU}$ heparin and $500 \mathrm{KIE}$ aprotinin per $\mathrm{ml}$ blood and centrifuged at $4^{\circ} \mathrm{C}$ within 30 minutes of collection. The plasma samples were stored at $-20^{\circ} \mathrm{C}$ until analysed for CCK. 
CCK DETERMINATIONS

Blood samples were thawed, centrifuged, and the plasma loaded onto C18 SEP-PAK cartridges for purification. Each cartridge was activated with $10 \mathrm{ml}$ of $100 \%$ acetonitrile and then washed with $10 \mathrm{ml}$ of $0 \cdot 1 \%$ acetic acid before plasma samples in $1 \mathrm{ml}$ portions were applied onto the cartridge. After washing with $5 \mathrm{ml}$ of $0.1 \%$ acetic acid, the samples were eluted by $6 \mathrm{ml}$ of a mixture containing $50 \%$ acetonitrile and $50 \%$ of $0 \cdot 1 \%$ acetic acid. The eluted samples were lyophilised and stored before further processing.

\section{HIGH PRESSURE LIQUID CHROMATOGRAPHY (HPLC)}

HPLC was used for separation of CCK from gastrin and also for separation of the different molecular forms of CCK (CCK-8, -33,39). Lyophilised samples were dissolved in a mixture of $50 \%$ distilled water and $50 \%$ of $0 \cdot 1 \%$ trifluoroacetic acid $(500 \mu \mathrm{l})$. Samples were injected on a TSK ODS-120 T column $(4.6 \times 250 \mathrm{~mm}, 5 \mu \mathrm{m}$ particle size), using an isocratic system of $34 \%$ acetonitrile in $0.1 \%$ trifluoroacetic acid at a flow rate of $1 \mathrm{ml} / \mathrm{min}$. With this HPLC system, good separation was obtained between non-sulphated and sulphated gastrin-17 and non-sulphated gastrin34 and the different molecular forms of CCK, CCK-8 was clearly separated from CCK33,39 which eluted closely. Fractions containing CCK-8 and CCK-33,39, respectively, were pooled and frozen and then lyophilised before later analysis by radioimmunoassay. The standards used were human gastrin-17 (non-sulphated, sulphated), human gastrin-34 (non-sulphated), CCK-8 (sulphated), from Universal Biological Ltd and porcine CCK-33 and CCK-39 (sulphated) were gifts from Professor V Mutt's laboratory.

\section{RADIOIMMUNOASSAY}

The lyophilised samples were reconstituted in the assay buffer $(0.02 \mathrm{M}$ veronal buffer) and CCK concentrations were determined by gastrin radioimmunoassay. The antiserum used was 2717, a gift from Professor J Rehfeld, Copenhagen, which reacts with the carboxy terminal pentapeptide common to both gastrin and CCK. This antiserum detects CCK-8 and CCK-33 with the same potency but showed $50 \%$ less affinity for CCK-39. CCK-8 was used as standard. The intra-assay and interassay coefficients of variation were $5 \%$ and $11 \%$, respectively. The sensitivity of the assay is $5 \mathrm{pmol} / \mathrm{l}$. CCK-8 and CCK-33 in amounts ranging from 0.05 to $0.4 \mathrm{pmol} / 1$ were added to $1 \mathrm{ml}$ dog plasma and submitted to SEP-PAK extraction and HPLC, about $65 \%$ of the material was recovered. For a more detailed description of the HPLC and radioimmunoassay methods see Lindén and Uvnäs-Moberg. ${ }^{2}$

\section{PRESENTATION OF DATA AND STATISTICAL}

CALCULATION

CCK concentrations are given as CCK-33,39 and CCK-8 separately as well as together (total CCK). Data are given as mean (SD).

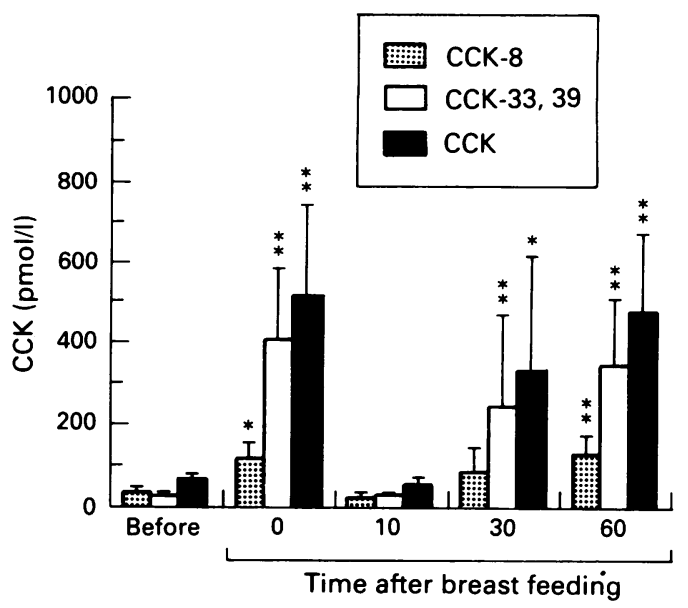

Mean (SD) plasma concentrations of CCK (pmoll) before, 0, 10, 30, and 60 minutes after breast feeding; $* p<0.05, * * p<0.01$.

Statistical analyses were performed using one factor analysis of variance. Correlations between variables were calculated using Spearman's correlation coefficient.

\section{Results}

The preprandial total CCK concentrations $(\mathrm{n}=12)$ was $68(17) \mathrm{pmol} / \mathrm{l}$. (CCK-33,39 and CCK-8 were 29 (11) pmol/l and 39 (10) $\mathrm{pmol} / 1$, respectively). The total CCK concentrations as well as CCK-33,39 were significantly raised immediately after the infant had stopped sucking $(p<0.01)$. A secondary rise was observed after 30 minutes $(p<0.05$ and $\mathrm{p}<0.01$ ) and 60 minutes later (figure). The postprandial pattern of CCK-8 was similar to that observed for CCK-33,39 but a significant rise was seen only at 60 minutes after breast feeding $(\mathrm{p}<0.01)$. The ratio between large $(\mathrm{CCK}-33,39)$ and small (CCK-8) CCK molecules was $1: 1$ before and 3:1 after breast feeding.

\section{CORRELATIONS}

Basal CCK concentrations (total as well as CCK-33,39) were inversely correlated to postnatal age (days 3-7): $r_{s}=-0.5, \mathrm{p}=0.05$ and $r_{s}=-0 \cdot 7, \mathrm{p}=0 \cdot 005$.

\section{Discussion}

Only a few reports on plasma concentrations of CCK in man have been published, the reason being methodological difficulties. Due to the chemical similarity between gastrin and CCK, most antisera cross react with gastrin and CCK and specific CCK antibodies have not been commercially available. In the present study we solved the problem by running each sample on HPLC thus separating gastrins from CCKs. The concentrations of CCK-8 and CCK-33, 39 were then measured separately with radioimmunoassay.

The basal concentrations recorded in newborns in the present study were about 10 times higher than those recorded with specific CCK radioimmunoassays in adults ${ }^{1}$ or in 9 month old children. ${ }^{13}$ With our method we have also recorded lower concentrations in 
adult subjects than in the newborns. ${ }^{14}$ Thus, we think that the CCK concentrations really are high in infancy-an assumption that is supported by inverse correlation between CCK concentrations and postnatal age during the first week of life.

The larger forms of CCK, that is CCK33,39, dominate CCK-8 after stimulation. This difference is not seen in adult women. ${ }^{14}$ Interestingly, the same preponderance for larger molecular forms is seen in infants also in the case of gastrin. ${ }^{15}$

From a physiological point of view, the most interesting finding is the biphasic release of CCK in response to breast feeding. This has not been shown earlier in children or in adults.

It is not likely that nutrients are responsible for the immediate postprandial CCK peak as plasma concentrations returned to basal 10 minutes later. We have shown that insulin concentrations increase ${ }^{7}$ and that the intragastric secretion of somatostatin decreases when infants suck a pacifier. ${ }^{8}$ As the release of both these hormones is vagally controlled, we concluded that the sucking stimulus triggers an activation of the vagal nerve. ${ }^{9}$ As the secretion of CCK is also under vagal influence ${ }^{5}$ the initial CCK response is more likely to have been caused by vagal mechanisms included by the sucking stimulus than by food itself.

The second more protracted increase of CCK concentrations, however, appearing 30-60 minutes after breast feeding was expected and likely to be a consequence of the presence of milk in the intestine. It is not yet known which factors in milk are responsible for the releasing effect. Fat, however, has been shown to be the most potent CCK releaser in adults. ${ }^{4}$

A very rapid growth and maturation of the gastrointestinal tract occur postnatally. The high basal and stimulated CCK concentrations may contribute to the postnatal development of the gastrointestinal tract as CCK exerts trophic effects, for example on the pancreas. ${ }^{10}$

It is well known that a newborn infant becomes not only satiated after feeding but also sedated and sleepy. Indeed, similar effects can be triggered by sucking of a pacifier alone. ${ }^{16} 17$ In this context it is of interest that the infants often were asleep postprandially and did not react to the venepuncture.

It is known from rat experiments that $\mathrm{CCK}$ causes satiety and a postprandial behaviour including sedation and sleep if injected intraperitoneally. The effect is mediated via afferent vagal fibres as it is abolished by vagotomy. ${ }^{11} 12$ It is therefore possible that the CCK released in the infant during breast feeding may via afferent vagal nerve activation influence central nervous system function in such a way that satiety, sedation, and sleepiness are induced. Other factors such as bombesin and gastric distention may of course also contribute to this effect.
Another interesting finding was that basal CCK concentrations were inversely related to age, that is the younger the infant the higher was its basal CCK concentration. Hypothetically this difference could be related to shorter feed intervals in the younger infants and therefore to persisting postprandial increases of CCK concentrations. However, no relationship between time for last feed and CCK concentrations could be demonstrated. Considering that CCK may as described above induce satiety and postprandial sedation, it is tempting to suggest that infants have high CCK concentrations in the first days of life in order to stay sedated and calm despite receiving very little food from their mothers.

We wish to thank the Swedish Medical Research Council, the First of May Flower Annual Campaign, Stiftelsen Clas Groschinskys Minnesfond, Stiftelsen Samariten, and ÅhlénStiftelsen for financial assistance.

1 Walsh JH, Lamers CB, Valenzuela JE. Cholecystokininoctapeptide-like immunoreactivity in human plasma. octapeptide-like immunoreactivity
Gastroenterology 1982;82:438-4.

2 Lindén A, Uvnäs-Moberg K. Plasma levels of cholecystokinin (CCK-8 and CCK-32-39) in response to feeding and during pregnancy in dogs. Scand $f$ Gastroenterol $1987 ; 22: 859-64$

3 Lindén-A, Uvnäs-Moberg K, Forsberg G, Bednar I, Södersten P. Plasma concentrations of cholecystokinin octapeptide and food intake in male rats treated with cholecystokinin octapeptide. $\mathcal{F}$ Endocrinol 1989;121: $59-65$.

4 Himeno S, Tarui S, Kanayama T, et al. Plasma cholecystokinin responses after ingestion of liquid meal and intraduodenal infusion of fat, amino acids, or hydrochloric acid in man: analysis with region specific radioimric acid in man: analysis with region specific
munoassay. Am $\mathcal{F}$ Gastroenterol 1983;78:703-7.

5 Schafmayer A, Nustede A, Pompino A, Köhler H. Vagal influence of cholecystokinin and neurotensin release in influence of cholecystokinin and neurotensin release

6 Lindén A, Eriksson M, Hansen S, Uvnäs-Moberg K. Suckling-induced release of cholecystokinin into plasma in the lactating rat: effects of abdominal vagotomy and lesions of central pathways concerned with milk ejection. $\mathcal{f}$ Endocrinol 1990;127:257-63.

7 Marchini G, Lagercrantz H, Feuerberg Y, Winberg J, Uvnäs-Moberg $\mathrm{K}$. The effect of non-nutritive suckling on plasma insulin, gastrin and somatostatin levels in infants. Acta Paediatr Scand 1987;76:573-8.

8 Widström A-M, Marchini G, Matthiesen A-S, Werner S, Winberg J, Uvnäs-Moberg K. Nonnutritive sucking in tube-fed preterm infants: effects of gastric motility and tube-fed preterm infants: effects of gastric motility and gastric contents of

9 Uvnäs-Moberg K, Widström A-M, Marchini G, Winberg $\mathrm{J}$. Release of GI hormones in mother and infant by sensory stimulation. Acta Paediatr Scand 1987;76:851-60.

10 Lankisch PG. Trophic effects of gastrointestinal hormones. In: Wolvey W, ed. Clinics in gastroenterology. Philadelphia: WB Saunders, 1980:773-84

11 Gibbs J, Young RC, Smith GP. Cholecystokinin decreases food intake in rats. Fournal of Comparative and Physiological Psychology 1973;84:488-95.

12 Antin J, Gibbs J, Holt J, Young RC, Smith GP. Cholecystokinin elicits the complete behavioural sequence of satiety in rats. Fournal of Comparative and sequence of satiety in rats. Fournal of

13 Salmenperä L, Perheentupa J, Siimes MA, Adrian TE, Bloom SR, Aynsley-Green A. Effects of feeding regimen Bloom SR, Aynsley-Green A. Effects of feeding regimen
on blood glucose levels and plasma concentrations of pancreatic hormones and gut regulatory peptides at 9 months of age: comparison between infants fed with milk formula and infants exclusively breast-fed from birth. f Pediatr Gastroenterol Nutr 1988;7:651-6.

14 Frick G, Bremme K, Sjögren C, Lindén A, Uvnäs-Moberg $\mathrm{K}$. Plasma levels of cholecystokinin and gastrin during the menstrual cycle and pregnancy. Acta Obstet Gynecol Scand 1990;69:317-20

15 Marchini G, Redham I, Uvnäs-Moberg K. Plasma gastrin34 increases during and immediately after breast feeding in 3-day old infants. 7 Pediatr Gastroenterol Nutr 1992; 14:140-5.

16 Kessen W, Leutzendorff AM. The effect of nonnutritive sucking on movement in the human newborn. Fournal of Comparative and Physiological Psychology 1963;56:69-72.

17 Field T, Goldson E. Pacifying effects of nonnutritive sucking on term and preterm neonates during heelstick procedures. Pediatrics 1984;84:1012-5. 